\title{
1 Zinc-embedded fabrics inactivate SARS-CoV-2 and influenza A virus
}

Vikram Gopal $^{1 *}$, Benjamin E. Nilsson-Payant ${ }^{2}$, Hollie French ${ }^{3}$, Jurre Y. Siegers ${ }^{4}$, Wai-shing

5 Yung $^{1}$, Matthew Hardwick ${ }^{5}$, Aartjan J.W. te Velthuis ${ }^{3^{*}}$

6

${ }^{1}$ Ascend Performance Materials, 1010 Travis Street, Suite 900, Houston, TX 77002, USA

${ }^{2}$ Department of Microbiology, Icahn School of Medicine at Mount Sinai, New York, NY 10029, USA

${ }^{3}$ Division of Virology, Department of Pathology, Addenbrooke's Hospital, University of Cambridge, Hills Road, CB2 2OQ, United Kingdom

${ }^{4}$ Department of Viroscience, Erasmus University Medical Centre, Rotterdam, the Netherlands

${ }^{5}$ ResInnova Laboratories, 8807 Colesville Rd, 3rd Floor, Silver Spring, MD 20910, USA

* address correspondence to: VGopal@ascendmaterials.com, ajwt6@cam.ac.uk

Key words: influenza, coronavirus, absorption, zinc, face mask 


\section{Abstract}

31 Infections with respiratory viruses can spread via liquid droplets and aerosols, and cause

32 diseases such as influenza and COVID-19. Face masks and other personal protective

33 equipment (PPE) can act as barriers that prevent the spread of respiratory droplets

34 containing these viruses. However, influenza A viruses and coronaviruses are stable for

35 hours on various materials, which makes frequent and correct disposal of these PPE

36 important. Metal ions embedded into PPE may inactivate respiratory viruses, but

37 confounding factors such as absorption of viruses make measuring and optimizing the

38 inactivation characteristics difficult. Here we used polyamide 6.6 (PA66) fibers that had zinc

39 ions embedded during the polymerisation process and systematically investigated if these

40 fibers can absorb and inactivate pandemic SARS-CoV-2 and influenza $A$ virus $\mathrm{H}_{1} \mathrm{~N}_{1}$. We

41 find that these viruses are readily absorbed by PA66 fabrics and inactivated by zinc ions

42 embedded into this fabric. The inactivation rate $\left(\mathrm{pfu} \cdot \mathrm{gram}^{-1} \cdot \mathrm{min}^{-1}\right.$ ) exceeds the number of

43 active virus particles expelled by a cough and supports a wide range of viral loads.

44 Moreover, we found that the zinc content and the virus inactivating property of the fabric

45 remain stable over 50 standardized washes. Overall, these results provide new insight into

46 the development of "pathogen-free" PPE and better protection against RNA virus spread. 


\section{Introduction}

50 Infections with influenza A viruses (IAV), influenza B viruses (IBV) and coronaviruses (CoV)

51 are a burden on our healthcare systems and economy. These respiratory RNA viruses

52 transmit through aerosols, liquid droplets and fomites and seasonal strains typically cause a

53 mild disease with symptoms including nasopharyngitis, fever, coughing, and headache.

54 Nevertheless, seasonal IAV and IBV result in 290,000-645,000 deaths every year and

55 billions of dollars in losses, in part due to increased hospitalizations and reduced work

56 efficiencies.

58 The impact of highly pathogenic and pandemic IAV and CoV strains is even more severe.

59 Over the past century, several pandemic influenza A virus (IAV) and severe acute 60 respiratory syndrome coronaviruses (SARS-CoV) strains have infected and killed millions of

61 people. Of particular importance were the $1918 \mathrm{H}_{1} \mathrm{~N}_{1}, 1957 \mathrm{H}_{2} \mathrm{~N}_{2}$, and the $1968 \mathrm{H}_{3} \mathrm{~N}_{2}$ 62 pandemic IAV strains, and the SARS-CoV-2 pandemic strain, the causative agent of COVID-

63 19. These viruses can cause viral pneumonia and make it easier for secondary bacterial 64 infections to take hold, increasing patient morbidity and mortality (1). Understanding how 65 we can efficiently prevent the spread of these viruses will be important for current and 66 future RNA virus outbreaks.

68 IAV is part of the Orthomyxoviridae, a family of negative-sense RNA viruses that are known 69 for their segmented RNA genomes. The virus particle is enveloped by a double-layered 70 membrane and contains multiple copies of the viral haemagglutinin ( $H A)$, matrix $2\left(\mathrm{M}_{2}\right)$, 71 and, neuraminidase (NA) proteins embedded in the membrane (2). The HA protein binds 72 sialic acid receptors on the outside of host cells, and fuses the viral membrane and the 
73 cellular plasma membrane, while the $M_{2}$ protein acts as proton channel that plays a role in

74 the activation of HA and release of the viral RNA genome into the host cell. The viral RNA

75 genome consists of eight segments that are encapsidated by the viral nucleoprotein (NP)

76 and RNA polymerase as ribonucleoprotein (RNP) complexes inside virus particles (3). After

77 viral transcription, protein synthesis, replication and virion formation, the NA protein is

78 required for the release of virus particles from the host cell.

80 SARS-CoV-2 belongs to the Coronaviridae, a family of positive-sense RNA viruses that

81 infect a wide range of vertebrates, including humans $(4,5)$. The SARS-CoV-2 virion consists

82 of a double-layered membrane and membrane proteins spike (S), envelope (E) and matrix

83 (M). The viral RNA genome is harboured inside the virus particle and encapsidated by the

84 viral nucleocapsid protein $(\mathrm{N})$. Infection of a host cell requires binding of the $\mathrm{S}$ protein to

85 the cellular receptor ACE2 (6). Following entry, the virus releases its viral RNA into the host

86 cell for viral protein synthesis and genome transcription and replication by the viral RNA

87 polymerase.

89 Various antivirals are currently available for the treatment of influenza and CoV infections,

90 including RNA polymerase inhibitors favipiravir and remdesivir (7-9). In addition, vaccines

91 are available or in development that target the HA or S protein to prevent infection and

92 spread of IAV and SARS-CoV-2 (10). Unfortunately, vaccines are not readily available for

93 emerging RNA viruses, and existing RNA viruses can become resistant against antivirals

94 and escape immune pressures due to their relatively high mutation rate $(11,12)$. Use of

95 personal protective equipment (PPE), such as face masks, is therefore recommended by

96 health organizations to prevent respiratory virus spread and several studies have supported 
97 their efficacy (13-15). However, opponents of the use of face masks have pointed to

98 complicating factors, including observations that respiratory viruses are stable for days to

99 hours on fabrics and that N95 respirators require careful decontamination to allow their re-

100 use (16-19). Further considerations are the additional environmental waste that disposable

101 face masks produce, the poor fit of some masks, and the potential health hazard that 102 discarded masks present $(20,21)$. Development of PPE that can trap and inactivate 103 respiratory viruses may help address some of these concerns or simplify their use in day-to104 daylife.

106 Previous research has shown that IAVs and CoVs can be inactivated by metal surfaces, such 107 as copper and zinc (22-24). While the exact underlying inactivation mechanisms are not 108 fully understood, evidence suggests that metal ions can induce RNA hydrolysis, membrane 109 destabilisation, or viral protein inactivation or degradation (25-27). So far, few studies have 110 investigated if metal ions embedded in fabrics can inactivate RNA viruses, in part because 111 absorbance and fabric density differences among fabrics present confounding factors that 112 the protocols approved for testing the inactivating properties of surfaces do not account 113 for.

115 To tackle some of these confounding issues, we here measured the ability of different 116 fabrics, such as cotton, polyamide 6.6 (PA66) and polypropylene (PPP), to trap $\mathrm{H}_{1} \mathrm{~N}_{1}$ IAV 117 and pandemic SARS-CoV-2, and we explore how we can remove these viruses from the 118 fabrics to test for inactivation. We find that cotton and PA66 readily absorb respiratory 119 viruses, and that zinc ions embedded in PA66-based fabrics resulted in approximately a 2$120 \log$ reduction in virus titer, which is more than sufficient to inactivate the number of 
121 infectious IAV virus particles ( 24 plaque forming units [pfu]) present in a cough (28). Virus

122 inactivation plateaued over time. Overall, these results provide new insight into the

123 protective properties of fabrics used for face masks and the development of "pathogen-

124 free" fabrics.

125

126

127 Results

128 Influenza virus absorbance by cotton, polypropylene and polyamide

129 Various studies have investigated the filtration properties of fabrics and have investigated

130 factors such as breathability, hydrophobicity and/or electrostaticity. Fabrics also have a

131 different weight per square meter $\left(\mathrm{gram} / \mathrm{m}^{2}\right)$ and different moisture retention abilities, with

132 cotton absorbing up to $500 \%$ of its weight and PA66 absorbing as little as $0.3 \%$ of its

133 weight, depending on environmental conditions (29). These different properties may affect

134 how fabrics trap and/or release aerosols or liquid droplets containing RNA viruses. For

135 instance, PPE with poor absorbing properties that become contaminated with viruses may

136 retain these viruses on their surface thus become a potential health hazard if not disposed

137 of properly. Presently, it is not fully understood how moisture retention is correlated with

138 virus particle absorption. To investigate this relationship, we added IAV strain A/WSN/33

$139\left(\mathrm{H}_{1} \mathrm{~N}_{1}\right)$ to International Antimicrobial Council (IAC) issued cotton, a textile PA66 fabric, or

140 PPP from a disposable type II 3-ply face mask (Fig. 1A). After a 30-min incubation at room

141 temperature, the fabrics were washed with PBS to remove unabsorbed virus (Fig. 1B). To

142 estimate the amount of remaining liquid on each fabric, each sample tube with fabric was

143 weighed and compared to its dry weight. As shown in Fig. 1 C and D, cotton and PA66

144 retained more liquid than PPP, both relative to the applied volume and the weight of the 
145 fabric. Subsequent analysis of the IAV titer in the input and fabric washes showed that the

146 cotton and woven PA66 fabrics readily absorbed the applied virus, while less virus was

147 observed by the PPP fabric (Fig. 1E, F), which is in line with the hydrophobicity of PPP (30).

148

A
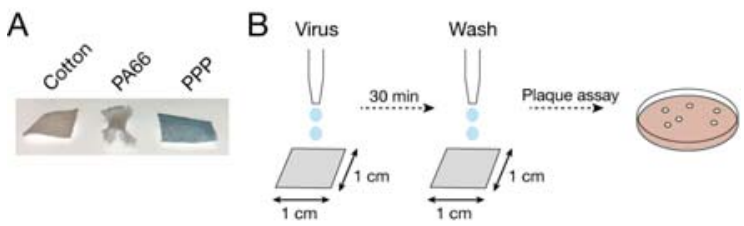

C
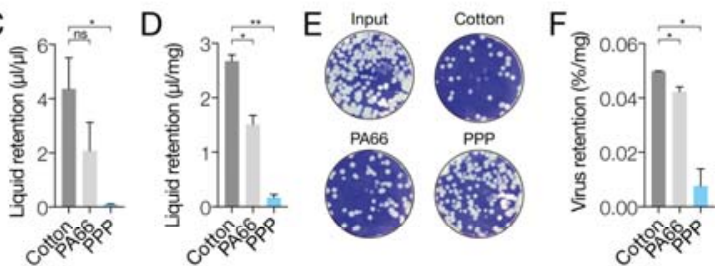

G
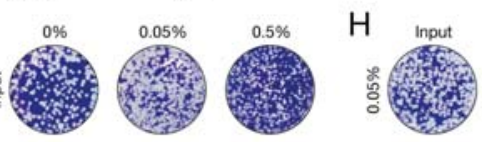

I Influenza A virus (H1N1)
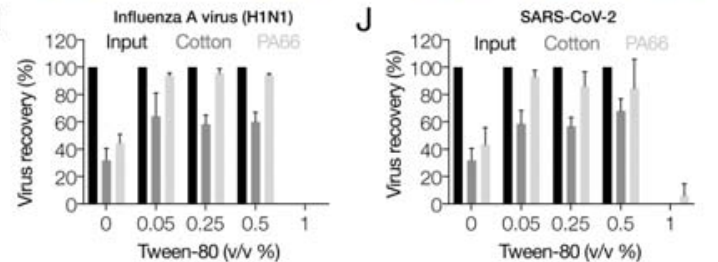

149

Figure 1. Absorption and release of IAV and SARS-CoV-2 from fabrics. A) Photographs of cotton

control, PA66 and polypropylene fabric samples. B) Schematic of experimental procedure for exposing

and isolating RNA virus from fabrics. C) Analysis of virus medium retention by fabrics per volume of

input medium. Values were obtained by weighing each fabric before and after addition of virus medium,

D) Analysis of virus medium retention by fabrics normalized by

dry weight of each fabric. Values were obtained by weighing each fabric before and after addition of virus medium, and after removal of the virus medium. E) Plaque assay of IAV present in virus medium after removal of the medium from each fabric. F) Quantitation of the amount of virus remaining on each fabric, normalized by the dry weight of each fabric. G) Effect of different tween-80 concentrations on IAV plaque assay read-out. $\mathrm{H}$ ) Effect of $0.05 \%$ tween-80 in PBS on the amount of virus released from each fabric. I) Quantitation of IAV titers after absorption of the virus to the fabrics and washing of the 
161 fabrics with PBS or PBS containing different concentrations of tween-80. J) Quantitation of SARS-CoV-2

162 titers after absorption of the virus to the fabrics and washing of the fabrics with PBS or PBS containing

163 different concentrations of tween-80. Error bars indicate standard deviation. Asterisk indicates p-value,

164 with * $p<0.05 * * p<0.005$, and ns $p>0.05$.

167 In order to remove IAV from the cotton and PA66 fabrics without inactivating the virus, we 168 added different concentrations of polysorbate-80 (tween-80) - a mild detergent that is also 169 used in IAV vaccine preparations - to the PBS wash buffer (Fig. 1G). We did not observe any 170 cytopathic effects of the detergent on the Madin-Darby Canine Kidney (MDCK) cells used

171 for the plaque assay, but did find that the presence of $0.05 \%-0.1 \%$ tween-80 increased the 172 apparent viral titer relative to infections in PBS (Fig. $1 G$ ), whereas $0.25-0.5 \%$ tween-80 173 reduced the apparent IAV plaque size (Fig. 1G). We found that $0.05 \%$ tween-80 succeeded 174 in recovering more than $94 \%$ of the virus from the PA66 woven fabric, whereas $61 \%$ was 175 removed from the cotton fabric (Fig. $1 \mathrm{H}$ and I). Higher concentrations, such as $1 \%$ tween176 8o, prevented IAV infection (Fig. 1l).

178 To confirm whether other viruses can be removed from cotton and woven PA66 as well, we 179 repeated the experiment with SARS-CoV-2. We found that over $92 \%$ of SARS-CoV-2 can be 180 recovered from the woven PA66 fabric using $0.05 \%$ tween- 80 , while up to $59 \%$ could be 181 recovered from the cotton fabric (Fig. IJ). Together, these results demonstrate that IAV 182 and SARS-CoV-2 are strongly absorbed by cotton and PA66, suggesting that these 183 materials would trap respiratory viruses inside face masks. At the same time, these findings 184 imply that PPP is poor at trapping respiratory viruses. Since IAV and SARS-CoV-2 can be 
185 removed from a PA66 fabric with a mild detergent, this protocol can be useful for testing

186 the inactivating properties of fabrics.

\section{Influenza virus is inactivated by zinc ions}

190 Copper and zinc surfaces or particles can inactivate IAV strains and seasonal CoV HCoV-

$191229 \mathrm{E}$, and PPP imbued with copper oxide can potentially inactivate IAV $(22,25,26,31)$. For

192 embedding into polymers, zinc ions provide benefits over copper ions as zinc has a much

193 higher propensity to ionize than copper, and thereby provides a much faster reaction

194 potential. Moreover, zinc oxide, which we embedded in the PA66 polymer used here, is

195 considered a Generally regarded as Safe (GRAS) compound by the FDA, which can speed

196 up the development process. Finally, zinc does not cause discolouration of the polymer or

197 fabric enabling a broader applicability. However, like copper, zinc ions are cytotoxic in

198 tissue culture (Fig. 2A), which confounds analysis of their effect on viral titers. We found

199 that addition of an equimolar concentration of EDTA following the virus incubation with

200 zinc ions (Fig. 2B) can efficiently chelate zinc ions and prevent cytotoxic effects (Fig. 2C).

201 EDTA alone does not have any cytotoxic effects and does not reduce viral titers (Fig. 2A).

202

A

A

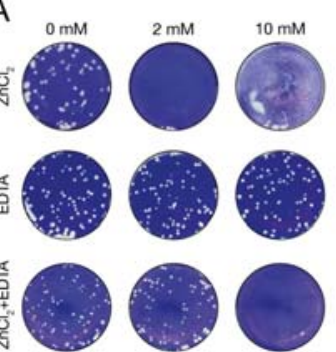

203
B

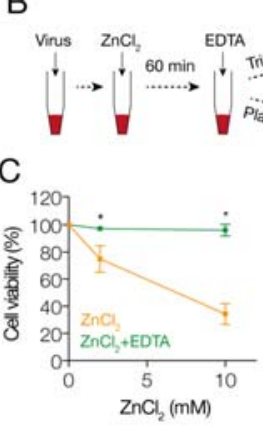

son

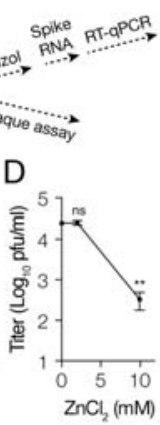

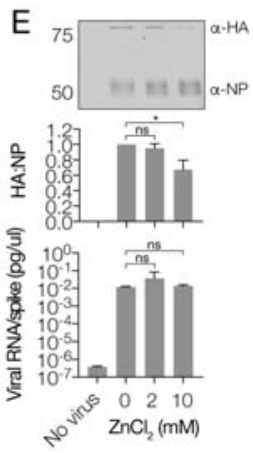


204 Figure 2. IAV is inactivated by zinc ions. A) Plaque assay showing the effect of different zinc chloride

205 and EDTA concentrations on IAV titers. B) Experimental approach for inactivating IAV with zinc ions and

206 neutralization of zinc ions using EDTA. C) Cytotoxicity analysis of zinc chloride and EDTA in MDCK cells.

207 D) IAV titers after exposure to zinc chloride and neutralization with EDTA as measured on MDCK cells. E)

208 Western blot IAV HA and NP protein levels after exposure to zinc chloride and neutralization with EDTA.

209 Upper panel shows quantitation of western signal and middle panel the western signal as detected with

210 LI-COR. Bottom panel shows NA segment RT-qPCR analysis of IAV virus after exposure to zinc chloride

211 and neutralization with EDTA. Error bars represent standard deviation. Asterisk indicates p-value, with *

$212 p<0.05$, ** $p<0.005$, and $n s p>0.05$.

214 To investigate if zinc ions can directly inactivate IAV, we incubated influenza virus with

215 varying concentrations of zinc chloride. After $60 \mathrm{~min}$, the reactions were stopped with an

216 equimolar amount of EDTA and subsequently diluted for virus titer determination by

217 plaque assay (Fig. 2B). As shown in Fig. 2D, we found that addition of zinc chloride resulted

218 in a significant reduction in the IAV titer. Previous research has shown that metal ions can

219 destabilize viral proteins (25). To gain more insight into the mechanism of virus

220 inactivation, viral protein levels in the zinc chloride-treated samples were analysed by

221 western blot. As shown in Fig. 2E, we found that in the presence of zinc chloride, HA levels

222 were reduced in a concentration-dependent manner, while NP levels did not diminish (Fig.

$2232 \mathrm{E})$. This result thus suggest that zinc ions may affect the IAV surface proteins more

224 significantly than the internal proteins. To test if IAV RNA levels were affected, we added a

225 120-nucleotide long spike RNA to each sample, extracted viral RNA, and performed reverse

226 transcriptions (RT) using a $3^{\text {? }}$ terminal NA primer. cDNA levels were next quantified using

227 quantitative polymerase chain reaction ( $q P C R$ ) of the NA gene-encoding segment and

228 normalized to the spike RNA level (Fig. 2E). No effect of zinc chloride on viral NA segment 
229 levels was found. Together, these results imply that zinc ions can inactivate an IAV $\left(\mathrm{H}_{1} \mathrm{~N}_{1}\right)$

230 strain by destabilization of the viral surface proteins.

231

232

233 Influenza and coronavirus strains are inactivated on fabrics containing zinc ions

234 The above results suggest that zinc ions can directly inactivate an IAV $\mathrm{H}_{1} \mathrm{~N}_{1}$ strain. To

235 investigate if these inactivating properties are also present when zinc ions are embedded in

236 a PA66 matrix, we used 0.4 gram of a textile PA66 fabric containing 328 ppm zinc ions

237 (equivalent to $2.5 \mathrm{mM}_{i}$ abbreviated as $\mathrm{KF}_{1}$ ). Incubation of $\mathrm{KF}_{1}$ with virus and washing of the

238 fabrics using a PBS buffer containing $0.05 \%$ tween-80 and $10 \mathrm{mM}$ EDTA (PBSTE; Fig. 3A),

239 resulted in an approximately 2 -log reduction of the IAV and SARS-CoV-2 titers compared to

240 a PA66 control fabric after $1 \mathrm{~h}$ (Fig. $3 \mathrm{~B}$ and C). To confirm that inactivation of these viruses

241 occurred on $\mathrm{KF}_{1}$, viral protein levels were analysed in the PBSTE wash eluate by western

242 blot (Fig. 3D and E). Any virus that remained in the fabric after extraction with PBSTE, was

243 lysed and extracted using Trizol and analyzed by western blot as well. Western blots

244 showed a reduction in the HA and S protein level in the virus eluate that was removed from

245 the KF1 fabric compared to the control fabric eluate for IAV and SARS-CoV-2, respectively

246 (Fig. 3D and E). The signal obtained from the virus that remained on each fabric after the

247 PBSTE extraction was close to background, in line with the observations in Fig. 1, and we

248 were only able to quantify the SARS-CoV-2 signal, but observed no statistically significant

249 difference. Overall, we conclude that inactivation of IAV and SARS-CoV-2 occurs on a fabric

250 embedded with zinc oxide, analogous to the previously observed effects of copper oxide

251 (31). To better investigate the rate of reduction, we incubated KF1 with virus for different

252 lengths of time and subtracted the absorbed virus titer in the negative control from the 
253 level of reduction in the $\mathrm{KF}_{1}$ fabric and fitted the data with a logarithmic equation (Fig. $3 \mathrm{~F}$ ).

254 A maximum reduction occurred between 30 seconds and 5 min of incubation, and the virus

255 titer reduction reached a plateau after approximately $50 \mathrm{~min}$.

256

A

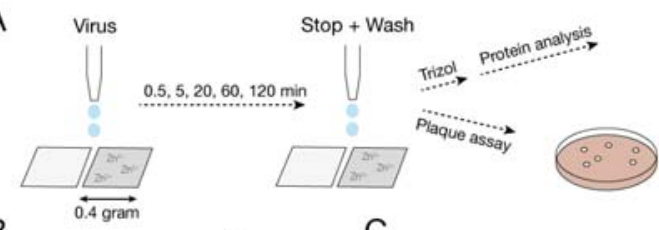

B

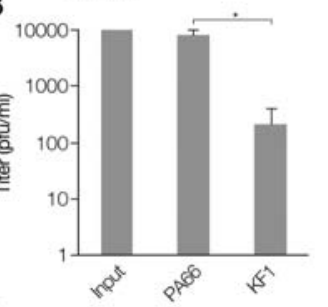

$\mathrm{D}$

Eluate Remaining

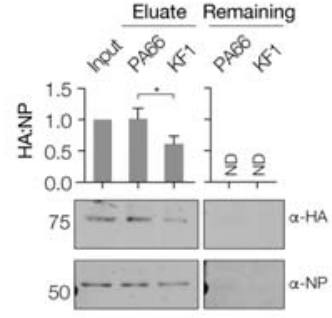

C

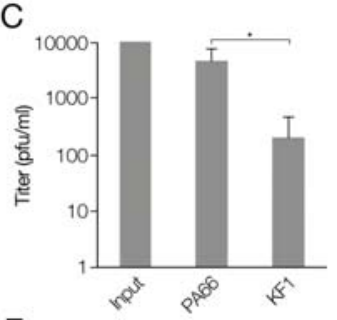

$\mathrm{E}$

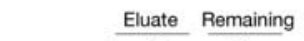

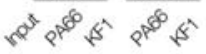

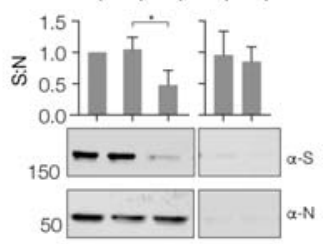

$\mathrm{F}$

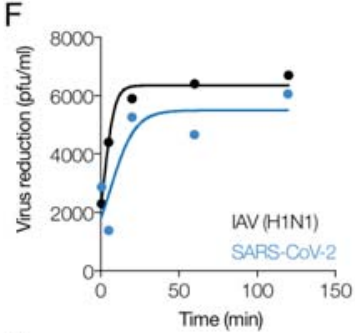

G

$\mathrm{H}$
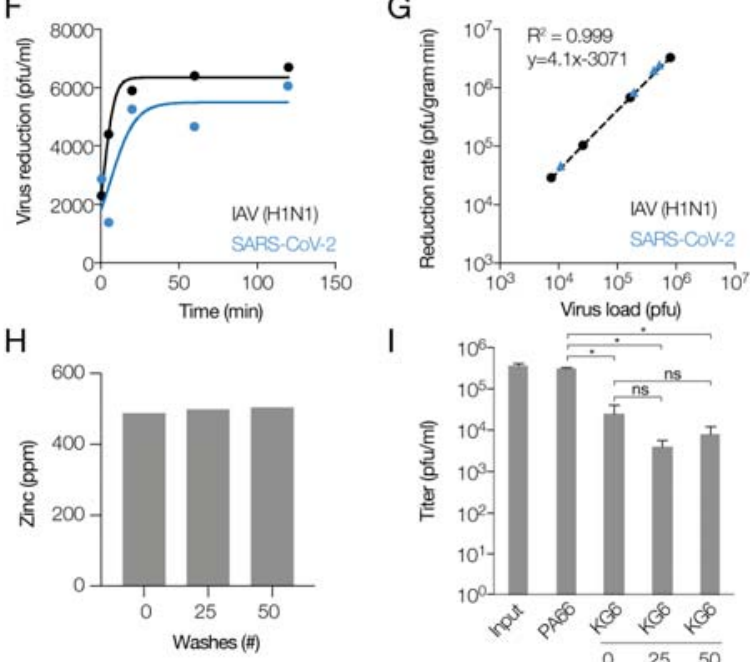

।

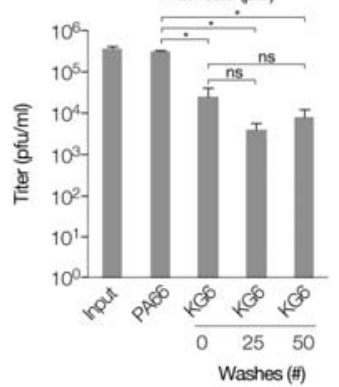

257

258 Figure 3. Inactivation of IAV and SARS-CoV-2 on fabrics. A) Schematic of testing procedure for fabrics

259 without or with embedded zinc oxide. B) IAV titer in input, or PA66 control or KF1 fabric eluates. C)

260 SARS-CoV-2 titer in input, or PA66 control or $\mathrm{KF}_{1}$ fabric eluates. One representative experiment is 
261 shown. D) Western blot analysis of IAV HA and NP protein levels after exposure of IAV to the KF1 or

262 control fabric. Both the virus that was removed (eluate) from each fabric with PBSTE as well as the virus

263 that remained on each fabric was analyzed. E) Western blot analysis of SARS-CoV-2 S and N protein

264 levels after exposure of virus to the $\mathrm{KF}_{1}$ or control fabric. Both the virus that was removed (eluate) from

265 each fabric with PBSTE as well as the virus that remained on each fabric was analyzed. F) Time course of

266 IAV or SARS-CoV-2 titer reduction by the $\mathrm{KF}_{1}$ fabric minus the titer reduction by the PA66 control

267 without embedded zinc. One representative time course is shown. Data were fit with logarithmic

268 equation. G) Reduction rate of IAV or SARS-CoV-2 titer after exposure to $\mathrm{KF}_{1}$ fabric. Data points were

269 obtained by from time courses experiments in which we varied the viral load and subsequently

270 estimated the maximum reduction rate (exponential phase) for each time course. Reduction was

271 normalized to $\mathrm{pfu} \cdot \mathrm{gram}^{-1} \cdot \mathrm{min}^{-1}$ using the dry fabric weight. IAV and SARS-CoV-2 data points were fit

272 with a linear line and no difference was observed between the two fits. $R^{2}$ for IAV fit is shown. H) Zinc

273 content in KG6 fabric after repeated washing according to the standardized home laundry test protocol

274 AATCC M6-2016. One representative experiment for one batch of fabric is shown. I) Reduction rate of

275 IAV titer after exposure to unwashed or washed KG6 fabric. Error bars represent standard deviation.

276 Asterisk indicates $p$-value, with * $p<0.05$ and $n s p>0.05$.

279 Inactivation of IAV and coronaviruses scales with virus load

280 To investigate the robustness and saturation level of the inactivation by fabrics containing

281 embedded zinc oxide, we next performed experiments with $\mathrm{KF}_{1}$ and varied the viral load

282 added to each fabric over a range of $10^{3}$ to $10^{7} \mathrm{pfu}$. The liquid volume applied to each fabric

283 was kept constant. After incubation for different periods of time, fabrics were washed with

284 PBSTE, virus titers estimated by plaque assay and the virus titer reduction rate calculated

285 based on the shortest incubation time. Reduction rates were subsequently normalized by

286 the dry weight of each fabric. As shown in Fig. $3 G$, the rate of reduction in virus titer (in 
$287 \mathrm{pfu} \cdot \mathrm{gram}^{-1} \cdot \mathrm{min}^{-1}$ ) scaled with virus load. On a log-log plot, the data could be fit with a linear

288 equation. To confirm the robustness of these findings, we performed the same

289 experiments with SARS-CoV-2, and found a similar behavior (Fig. $3 \mathrm{H})$.

290 To investigate if fabrics constructed from fibers containing zinc ions maintain their

291 zinc oxide content after washing, a KF1 fabric with $500 \mathrm{ppm}$ zinc ions (equivalent to $5.3 \mathrm{mM}$;

292 internal code KG6) was washed 25 or 50 times using the standardized home laundry test

293 protocol AATCC M6-2016. Subsequent analysis of the zinc content after washing revealed

294 that the zinc content remained relatively constant in the PA66 fabrics for up to 50 washes

295 (Fig. $3 \mathrm{H}$ ). We next confirmed if these washed fabrics were still able to reduce virus titers and

296 incubated $0.4 \mathrm{~g}$ of unwashed or washed fabric with a fixed amount of IAV and removed

297 inactivated virus with PBSTE. Analysis of the virus titers showed that both washed fabrics

298 were able reduce the IAV titer by approximately 2-logs (Fig. 3l). Overall, these results 299 suggest that the PA66 fabric containing zinc can inactivate both IAV and SARS-CoV-2 and 300 that this property is retained after 50 washes.

301

\section{Discussion}

303 Infections with respiratory RNA viruses cause regular seasonal epidemics and occasional

304 pandemics, and thus present a severe burden on our personal health, healthcare systems,

305 and economy. While seasonal respiratory viruses - including over 160 different rhinoviruses,

306 human CoVs strains NL63, OC43, HKU1 and 229E, influenza $A, B$ and $C$ viruses, human

307 respiratory syncytial virus, human parainfluenza viruses, and human metapneumovirus -

308 typically cause mild disease, IAV and CoVs have also been associated with zoonotic

309 outbreaks and lethal pandemics. Vaccines and antivirals are available or in development for

310 various respiratory viruses, but the appearance of resistance to antivirals or vaccines is a 
311 known or potential problem. In the absence of new vaccines or antivirals, one way to fight

312 RNA viruses is to limit respiratory virus spread through efficient PPE.

313

314 To better understand how respiratory RNA viruses are absorbed and inactivated on fabrics,

315 we here added IAV and SARS-CoV-2 to cotton, PA66, and PPP. We find strong absorption

316 by cotton and PA66 in the standard laboratory buffer PBS, and that addition of tween-80

317 results in efficient virus release from PA66, but not from cotton. A previous clinical trial

318 found that cotton masks with strong absorbing properties may be associated with a higher

319 risk of infection when reused and our finding that cotton does not release IAV or SARS-

320 CoV-2 efficiently after washing is in line with this observation (16). By contrast, virus

321 retention on PPP, which is used for the construction of disposable 3-ply masks, is poor, in

322 line with its hydrophobic properties (30). This result implies that respiratory viruses remain

323 on the surface of these masks and together with findings that SARS-CoV-2 can survive on

324 various surfaces for several hours to days, and even 7 days on PPP-based surgical face

325 masks (19, 32), PPP-based masks may increase the risk of infection if not handled and

326 disposed of properly. However, PPP has of course alternative properties, such as good

327 breathability, filtration, and electrostatic properties and will thus has a purpose in the right

328 situation.

330 The use of any face mask or other PPE, even if used temporarily but correctly to prevent

331 spread, is better than wearing no face mask as it may reduce the risk of infection with

332 respiratory viruses $(13-15)$. However, the above considerations suggest that there is a

333 potential for the use of pathogen-inactivating PPE, i.e. fabrics that can both absorb as well

334 as inactivate viruses. In particular the use of zinc and copper ions in PPE is promising in this 
335 regard, as these metals can inactivate IAV and SARS-CoV-2 (Fig. 2, 3) (22, 24, 26). Using a

336 PA66-based fabric from which we could easily remove absorbed virus with a mild detergent

337 (Fig. 1), we tested the effect of zinc ions embedded in a matrix on the infectiousness of IAV

338 and pandemic SARS-CoV-2. We consistently found a rapid reduction in the titer of all

339 viruses tested and at viral loads that far exceed the number of infectious IAV particles

340 present in a cough (Fig. 3). After washing the fabrics using a standardized protocol, both

341 the zinc content as well as the inactivating properties of the PA66 fabric were retained,

342 suggesting that this fabric is reusable at least 50 times. This property may be of particular

343 importance for designing reusable PPE that could help reduce environmental waste, virus

344 transmission, and costs.

346 We also investigated the mechanism by which zinc ions inactivate IAV and SARS-CoV-2.

347 RT-qPCR analysis showed no significant reduction in viral RNA integrity after treatment

348 with zinc ions. By contrast, analysis of the stability of the viral surface and capsid proteins

349 revealed a reduced stability of the virus surface proteins HA and S, for IAV and SARS-CoV-

3502 , respectively, after exposure to zinc ions, while no effect on the internal nucleoprotein or

351 nucleocapsid proteins was detected. We observed a similar altered surface protein to

352 nucleoprotein ratio after exposure to the zinc containing PA66 fabric KF1. Together, these

353 results suggest that the reduction in virus titer after exposure to zinc ions derives from

354 inactivation of the viral surface proteins. This is in line with previous research using copper

355 ions (25). Research has shown that zinc and copper ions can also induce oxidative reactions,

356 inactivation of the viral proton channels, or viral membrane destabilization and we cannot

357 exclude that these processes may play a role in the inactivation as well $(27,33,34)$. 
359 Overall, these results strongly suggest that virus inactivating fabrics can offer enhanced

360 safety over widely used cotton and PPP-based PPE. Our findings may therefore be

361 important for health care workers who are exposed to infected patients for prolonged

362 periods, people with underlying risk factors needing additional protection, and people who

363 need to frequently remove their PPE.

366 Methods

367

\section{Influenza viruses and cells}

369 MDCK cells were originally sources from ATCC. Influenza A/WSN/33 $\left(\mathrm{H}_{1} \mathrm{~N}_{1}\right)$ virus was

370 rescues from plasmids (35) and grown on MDCK cells in Minimal Essential Medium (MEM)

371 containing $0.5 \%$ foetal bovine serum (FBS) at $37{ }^{\circ} \mathrm{C}$ and $5 \% \mathrm{CO}_{2}$. Plaque assays were

372 performed on $100 \%$ confluent MDCK cells in MEM containing $0.5 \%$ FBS with a $1 \%$ agarose

373 overlay. Ten-fold virus dilutions were grown under a $1 \%$ agarose in MEM containing $0.5 \%$

374 FBS overlay for 2 days at $37^{\circ} \mathrm{C}$. Cell viability was measured using a CellTiter Blue assay

375 (Promega).

377 Coronaviruses and cells

378 SARS-CoV-2 (Bavpat-1 and USA-WA1/2020) were grown on African Green Monkey kidney

379 epithelial Vero-E6 cells in Dulbecco's Minimal Essential Medium (DMEM) supplemented

380 with 10\% FBS. For plaque assay analysis, Vero-E6 cells were seeded in 12-well plates and

381 infected at 100\% confluency. Ten-fold virus dilutions were grown under a $1 \%$ agarose 
overlay in DMEM containing $0.5 \%$ FBS for 2 days at $37^{\circ} \mathrm{C}$. Experiments were performed in a

383 BSL3 lab according to approved biosafety standards.

\section{Fabrics}

386 Fabric samples were cut by cleaned scissors. The cotton fabric was issued and certified by 387 the IAC (lot number IACVC01012020). The PA66 fabrics with zinc ions (Microban Additive 388 Zo7; EPA Reg. No. 42182-8) and a control fabric without zinc ions were produced and 389 provided by Ascend Performance Materials. The PPP disposable type II 3-ply face mask 390 (Medical Products Co, Ltd) was BS EN14638:2019 type II compliant. PA66 fabrics were 391 washed according to the standardized home laundry test protocol AATCC M6-2016. 392 Inductively coupled plasma (ICP) analysis was used to determine the zinc content after 393 fabric washing.

\section{Virus absorption and extraction}

396 To test the ability of fabrics to reduce viral titres, we used a modified ISO 18184 protocol.

397 Briefly, $100 \mu \mathrm{l}$ of IAV strain A/WSN/33 $\left(\mathrm{H}_{1} \mathrm{~N}_{1}\right)$ was applied in 5-10 $\mu$ l droplets to cotton, a

398 textile PA66 fabric, or PPP cut from medical grade face masks. The size of the fabrics varied 399 from $1 \mathrm{~cm}^{2}$ to 0.4 gram, as indicated in the figures. After an incubation at room 400 temperature as indicated, the fabrics were washed with PBS, PBS containing tween-80, or 401 PBS containing $0.05 \%$ tween-80 and $10 \mathrm{mM}$ EDTA through vortexing. After virus removal, 1 $402 \mathrm{ml}$ of Trizol (Invitrogen) was added to each fabric to extract remaining viral protein and 403 RNA. Experiments were performed in triplicate, unless noted otherwise. Data was analysed 404 in Graphpad Prism 8 using 1-way ANOVA. 


\section{RT-qPCR and western blot}

407 RNA extraction from Trizol was performed as described previously(36), while protein was

408 extracted from the interphase using isopropanol precipitation(37). Precipitated protein was

409 washed in ethanol, resuspended in 5x SDS-PAGE loading buffer, sonicated for 10 seconds,

410 and boiled for 10 min before $8 \%$ SDS-PAGE analysis. Western blot was performed using

411 antibodies directed against IAV HA (Invitrogen, PA5-34929) and NP (GeneTex, GTX125989)

412 and SARS-CoV-2 S (Abcam ab272504) and N (GeneTex, GTX632269). Membranes were

413 washed in TBS containing $0.1 \%$ tween-20. Spike RNA was purchased from IDT and had the

414 sequence 5?

415 AGUAGAAACAAGGCGGUAGGCGCUGUCCUUUAUCCAGACAACCAUUACCUGUCCACACA

416 AUCUGCCCUUUCGAAAGAUCCCAACGAAAAGAGAGACCACAUGGUCCUUCCUGCUUUU

417 GCU-3?]. Isolated RNA was reverse transcribed using SuperScript III and a primer binding to

418 the $3^{\prime}$ end of the NA segment (36). qPCR was performed as described previously (36). Data

419 was analysed in Graphpad Prism 8 using one-way ANOVA with multiple corrections.

\section{Acknowledgments}

423 We thank Shanaka Rodrigo, Natasha Virjee, Chris Hsiung, and Benjamin Tenoever

424 for helpful discussions, materials and information. AtV is supported by joint

425 Wellcome Trust and Royal Society grant 206579/Z/17/Z and the National Institutes of 426 Health grant R21Al147172. 
430 This study was funded in part by Ascend Performance Materials. VG and W-sY are

431 employed by Ascend Performance Materials. MH is employed by ResInnova and hired by

432 Ascend Performance Materials to perform experiments and analyze the data. Icahn School

433 of Medicine at Mount Sinai and University of Cambridge received consultancy fees from

434 Ascend Performance Materials for experimental work and data analysis.

\section{References}

438 1. McCullers JA. 2014. The co-pathogenesis of influenza viruses with bacteria in the

439 lung. Nat Rev Microbiol 12:252-62.

440 2. Hutchinson EC, Charles PD, Hester SS, Thomas B, Trudgian D, Martinez-Alonso M,

441 Fodor E. 2014. Conserved and host-specific features of influenza virion architecture.

$442 \quad$ Nat Commun 5:4816.

443 3. Te Velthuis AJ, Fodor E. 2016. Influenza virus RNA polymerase: insights into the

444 mechanisms of viral RNA synthesis. Nat Rev Microbiol 14:479-93.

445 4. Zhu N, Zhang D, Wang W, Li X, Yang B, Song J, Zhao X, Huang B, Shi W, Lu R, Niu P,

446 Zhan F, Ma X, Wang D, Xu W, Wu G, Gao GF, Tan W, China Novel Coronavirus I,

447 Research T. 2020. A Novel Coronavirus from Patients with Pneumonia in China, 2019.

$448 \quad$ N Engl J Med 382:727-733.

449 5. Coronaviridae Study Group of the International Committee on Taxonomy of V. 2020.

$450 \quad$ The species Severe acute respiratory syndrome-related coronavirus: classifying 2019-

451 nCoV and naming it SARS-CoV-2. Nat Microbiol 5:536-544. 
452 6. Letko M, Marzi A, Munster V. 2020. Functional assessment of cell entry and receptor usage for SARS-CoV-2 and other lineage B betacoronaviruses. Nat Microbiol 5:562-

454 569.

455 7. Goldhill DH, Te Velthuis AJW, Fletcher RA, Langat P, Zambon M, Lackenby A, Barclay WS. 2018. The mechanism of resistance to favipiravir in influenza. Proc Natl Acad Sci U S A 115:11613-11618.

458 8. Pruijssers AJ, George AS, Schafer A, Leist SR, Gralinksi LE, Dinnon KH, 3rd, Yount BL, 459 Agostini ML, Stevens LJ, Chappell JD, Lu X, Hughes TM, Gully K, Martinez DR, Brown AJ, Graham RL, Perry JK, Du Pont V, Pitts J, Ma B, Babusis D, Murakami E, Feng JY, Inhibits SARS-CoV-2 in Human Lung Cells and Chimeric SARS-CoV Expressing the

9. Williamson BN, Feldmann F, Schwarz B, Meade-White K, Porter DP, Schulz J, van Saturday G, Bosio CM, Anzick S, Barbian K, Cihlar T, Martens C, Scott DP, Munster VJ, de Wit E. 2020. Clinical benefit of remdesivir in rhesus macaques infected with SARSCoV-2. Nature 585:273-276.

10. van Riel D, de Wit E. 2020. Next-generation vaccine platforms for COVID-19. Nat Mater 19:810-812.

471 11. Eckerle LD, Becker MM, Halpin RA, Li K, Venter E, Lu X, Scherbakova S, Graham RL, PLoS Pathog 6:e1000896. 
475 12. Cheung PP, Rogozin IB, Choy KT, Ng HY, Peiris JS, Yen HL. 2015. Comparative mutational analyses of influenza A viruses. RNA 21:36-47.

477 13. Gandhi M, Beyrer C, Goosby E. 2020. Masks Do More Than Protect Others During COVID-19: Reducing the Inoculum of SARS-CoV-2 to Protect the Wearer. J Gen Intern Med doi:10.1007/s11606-020-06067-8.

14. Jefferson T, Del Mar CB, Dooley L, Ferroni E, Al-Ansary LA, Bawazeer GA, van Driel or reduce the spread of respiratory viruses. Cochrane Database Syst Rev doi:10.1002/14651858.CD006207.pub4:CD006207.

15. Wu J, Xu F, Zhou W, Feikin DR, Lin CY, He X, Zhu Z, Liang W, Chin DP, Schuchat A. 2004. Risk factors for SARS among persons without known contact with SARS patients, Beijing, China. Emerg Infect Dis 10:210-6. masks in healthcare workers. BMJ Open 5:e006577.

490 17. Wang Y, Tian H, Zhang L, Zhang M, Guo D, Wu W, Zhang X, Kan GL, Jia L, Huo D, Liu B, transmission of SARS-CoV-2 in households by face mask use, disinfection and social

494 18. Fischer RJ, Morris DH, van Doremalen N, Sarchette S, Matson MJ, Bushmaker T, 
498 19. Chin AWH, Chu JTS, Perera MRA, Hui KPY, Yen HL, Chan MCW, Peiris M, Poon LLM. 2020. Stability of SARS-CoV-2 in different environmental conditions. Lancet Microbe 1:e10.

501 20. Prata JC, Silva ALP, Walker TR, Duarte AC, Rocha-Santos T. 2020. COVID-19 Pandemic Repercussions on the Use and Management of Plastics. Environ Sci Technol 54:77607765.

504 21. Adyel TM. 2020. Accumulation of plastic waste during COVID-19. Science 369:13141315.

506 22. Noyce JO, Michels H, Keevil CW. 2007. Inactivation of influenza A virus on copper $507 \quad$ versus stainless steel surfaces. Appl Environ Microbiol 73:2748-50.

508 23. Biryukov J, Boydston JA, Dunning RA, Yeager JJ, Wood S, Reese AL, Ferris A, Miller D, 509 Weaver W, Zeitouni NE, Phillips A, Freeburger D, Hooper I, Ratnesar-Shumate S, 510 Yolitz J, Krause M, Williams G, Dawson DG, Herzog A, Dabisch P, Wahl V, Hevey MC, Altamura LA. 2020. Increasing Temperature and Relative Humidity Accelerates Inactivation of SARS-CoV-2 on Surfaces. mSphere 5.

513 24. Warnes SL, Little ZR, Keevil CW. 2015. Human Coronavirus 229E Remains Infectious on Common Touch Surface Materials. mBio 6:e01697-15.

515 25. Fujimori Y, Sato T, Hayata T, Nagao T, Nakayama M, Nakayama T, Sugamata R, Suzuki

516 K. 2012. Novel antiviral characteristics of nanosized copper(I) iodide particles

517 showing inactivation activity against 2009 pandemic H1N1 influenza virus. Appl $518 \quad$ Environ Microbiol 78:951-5.

$51926 . \quad$ Imai K, Ogawa H, Bui VN, Inoue H, Fukuda J, Ohba M, Yamamoto Y, Nakamura K. 520 2012. Inactivation of high and low pathogenic avian influenza virus H5 subtypes by 521 copper ions incorporated in zeolite-textile materials. Antiviral Res 93:225-233. 
522 27. Binder H, Arnold K, Ulrich AS, Zschornig O. 2001. Interaction of Zn2+ with

523 phospholipid membranes. Biophys Chem 90:57-74.

524 28. Lindsley WG, Noti JD, Blachere FM, Thewlis RE, Martin SB, Othumpangat S,

$525 \quad$ Noorbakhsh B, Goldsmith WT, Vishnu A, Palmer JE, Clark KE, Beezhold DH. 2015.

526 Viable influenza A virus in airborne particles from human coughs. J Occup Environ

$527 \quad$ Hyg 12:107-13.

528 29. Cruz J, Leitao AC, Silveira D, Pichandi S, Pinto M, Fangueiro R. 2017. Study of

529 moisture absorption characteristics of cotton terry towel fabrics. Procedia

$530 \quad$ Engineering 200:389-398.

531 30. Erbil HY, Demirel AL, Avci Y, Mert O. 2003. Transformation of a simple plastic into a $532 \quad$ superhydrophobic surface. Science 299:1377-80.

533 31. Borkow G, Zhou SS, Page T, Gabbay J. 2010. A novel anti-influenza copper oxide

$534 \quad$ containing respiratory face mask. PLoS One 5:e11295.

535 32. van Doremalen N, Bushmaker T, Morris DH, Holbrook MG, Gamble A, Williamson BN,

536 Tamin A, Harcourt JL, Thornburg NJ, Gerber SI, Lloyd-Smith JO, de Wit E, Munster VJ.

537 2020. Aerosol and Surface Stability of SARS-CoV-2 as Compared with SARS-CoV-1. N

$538 \quad$ Engl J Med 382:1564-1567.

539 33. Gandhi CS, Shuck K, Lear JD, Dieckmann GR, DeGrado WF, Lamb RA, Pinto LH. 1999.

$540 \mathrm{Cu}(\mathrm{II})$ inhibition of the proton translocation machinery of the influenza A virus M2

$541 \quad$ protein. J Biol Chem 274:5474-82.

542 34. Okada A, Miura T, Takeuchi H. 2003. Zinc- and pH-dependent conformational

543 transition in a putative interdomain linker region of the influenza virus matrix

$544 \quad$ protein M1. Biochemistry 42:1978-84. 
545 35. Fodor E, Devenish L, Engelhardt OG, Palese P, Brownlee GG, Garcia-Sastre A. 1999.

546 Rescue of Influenza A Virus from Recombinant DNA. Journal of Virology 73:9679-

$547 \quad 9682$.

548 36. Te Velthuis AJW, Long JC, Bauer DLV, Fan RLY, Yen HL, Sharps J, Siegers JY, Killip MJ,

549 French H, Oliva-Martin MJ, Randall RE, de Wit E, van Riel D, Poon LLM, Fodor E.

550 2018. Mini viral RNAs act as innate immune agonists during influenza virus infection.

$551 \quad$ Nat Microbiol 3:1234-1242.

552 37. Simoes AE, Pereira DM, Amaral JD, Nunes AF, Gomes SE, Rodrigues PM, Lo AC,

553 D'Hooge R, Steer CJ, Thibodeau SN, Borralho PM, Rodrigues CM. 2013. Efficient

554 recovery of proteins from multiple source samples after TRIzol((R)) or TRIzol((R))LS

$555 \quad$ RNA extraction and long-term storage. BMC Genomics 14:181.

556

557

558 Figure legends

559

560 Figure 1. Absorption and release of IAV and SARS-CoV-2 from fabrics. A) Photographs of

561 cotton control, PA66 and polypropylene fabric samples. B) Schematic of experimental

562 procedure for exposing and isolating RNA virus from fabrics. C) Analysis of virus medium

563 retention by fabrics per volume of input medium. Values were obtained by weighing each

564 fabric before and after addition of virus medium, and after removal of the virus medium. D)

565 Analysis of virus medium retention by fabrics normalized by dry weight of each fabric.

566 Values were obtained by weighing each fabric before and after addition of virus medium,

567 and after removal of the virus medium. E) Plaque assay of IAV present in virus medium

568 after removal of the medium from each fabric. F) Quantitation of the amount of virus 
569 remaining on each fabric, normalized by the dry weight of each fabric. G) Effect of different

570 tween-80 concentrations on IAV plaque assay read-out. H) Effect of $0.05 \%$ tween-80 in PBS

571 on the amount of virus released from each fabric. I) Quantitation of IAV titers after

572 absorption of the virus to the fabrics and washing of the fabrics with PBS or PBS containing

573 different concentrations of tween-80. J) Quantitation of SARS-CoV-2 titers after absorption

574 of the virus to the fabrics and washing of the fabrics with PBS or PBS containing different

575 concentrations of tween-8o. Error bars indicate standard deviation. Asterisk indicates p-

576 value, with * $p<0.05$, ** $p<0.005$, and $n s p>0.05$

579 Figure 2. IAV is inactivated by zinc ions. A) Plaque assay showing the effect of different

580 zinc chloride and EDTA concentrations on IAV titers. B) Experimental approach for 581 inactivating IAV with zinc ions and neutralization of zinc ions using EDTA. C) Cytotoxicity 582 analysis of zinc chloride and EDTA in MDCK cells. D) IAV titers after exposure to zinc 583 chloride and neutralization with EDTA as measured on MDCK cells. E) Western blot IAV HA 584 and NP protein levels after exposure to zinc chloride and neutralization with EDTA. Upper 585 panel shows quantitation of western signal and middle panel the western signal as detected 586 with LI-COR. Bottom panel shows NA segment RT-qPCR analysis of IAV virus after 587 exposure to zinc chloride and neutralization with EDTA. Error bars represent standard 588 deviation. Asterisk indicates $p$-value, with $* p<0.05, * * p<0.005$, and ns $p>0.05$.

591 Figure 3. Inactivation of IAV and SARS-CoV-2 on fabrics. A) Schematic of testing 592 procedure for fabrics without or with embedded zinc oxide. B) IAV titer in input, or PA66 
593 control or $\mathrm{KF}_{1}$ fabric eluates. C) SARS-CoV-2 titer in input, or PA66 control or $\mathrm{KF}_{1}$ fabric

594 eluates. One representative experiment is shown. D) Western blot analysis of IAV HA and

595 NP protein levels after exposure of IAV to the KF1 or control fabric. Both the virus that was

596 removed (eluate) from each fabric with PBSTE as well as the virus that remained on each

597 fabric was analyzed. E) Western blot analysis of SARS-CoV-2 S and N protein levels after

598 exposure of virus to the $\mathrm{KF}_{1}$ or control fabric. Both the virus that was removed (eluate) from

599 each fabric with PBSTE as well as the virus that remained on each fabric was analyzed. F)

600 Time course of IAV or SARS-CoV-2 titer reduction by the $\mathrm{KF}_{1}$ fabric minus the titer

601 reduction by the PA66 control without embedded zinc. One representative time course is

602 shown. Data were fit with logarithmic equation. G) Reduction rate of IAV or SARS-CoV-2

603 titer after exposure to $\mathrm{KF}_{1}$ fabric. Data points were obtained by from time courses

604 experiments in which we varied the viral load and subsequently estimated the maximum

605 reduction rate (exponential phase) for each time course. Reduction was normalized to

$606 \mathrm{pfu} \cdot \mathrm{gram}^{-1} \cdot \mathrm{min}^{-1}$ using the dry fabric weight. IAV and SARS-CoV-2 data points were fit with

607 a linear line and no difference was observed between the two fits. $R^{2}$ for IAV fit is shown. $H$ )

608 Zinc content in KG6 fabric after repeated washing according to the standardized home

609 laundry test protocol AATCC M6-2016. One representative experiment for one batch of

610 fabric is shown. I) Reduction rate of IAV titer after exposure to unwashed or washed KG6

611 fabric. Error bars represent standard deviation. Asterisk indicates p-value, with * $p<0.05$

612 and $n s p>0.05$. 